\title{
Efficacy of Osimertinib Plus Bevacizumab In Glioblastoma Patients With Simultaneous EGFR Amplification And EGFRvIll Mutation
}

Daniel Jaramillo-Velásquez ( $\nabla$ djaramillomd@gmail.com )

Fundacion Santa Fe de Bogota https://orcid.org/0000-0002-3261-7430

Andrés F. Cardona

Clinica del Country

Alejandro Ruiz-Patiño

Universidad El Bosque

Carolina Polo

Universidad El Bosque

Enrique Jiménez

Fundacion Santa Fe de Bogotá: Fundacion Santa Fe de Bogota

Fernando Hakim

Fundacion Santa Fe de Bogotá: Fundacion Santa Fe de Bogota

Diego F Gómez

Fundacion Santa Fe de Bogotá: Fundacion Santa Fe de Bogota

Juan Fernando Ramón

Fundacion Santa Fe de Bogotá: Fundacion Santa Fe de Bogota

Hernando Cifuentes

Clinica del Country

Juan Armando Mejía

Fundacion Santa Fe de Bogotá: Fundacion Santa Fe de Bogota

Fernando Salguero

Fundacion Santa Fe de Bogotá: Fundacion Santa Fe de Bogota

\section{Camila Ordoñez}

Universidad El Bosque

\section{Álvaro Muñoz}

Fundacion Santa Fe de Bogotá: Fundacion Santa Fe de Bogota

\section{Sonia Bermúdez}

Fundacion Santa Fe de Bogotá: Fundacion Santa Fe de Bogota

Nicolas Useche

Fundacion Santa Fe de Bogotá: Fundacion Santa Fe de Bogota

Diego Pineda

Clinica del Country

\section{Luisa Ricaurte}

Mayo Clinic Rochester: Mayo Clinic Minnesota 


\section{Zyanya Lucia Zatarain-Barrón}

Mexico National Cancer Institute: Instituto Nacional de Cancerologia

July Rodríguez

Universidad El Bosque

Jenny Avila

Universidad El Bosque

\section{Leonardo Rojas}

Clinica del Country

\section{Elvira Jaller}

Universidad El Bosque

\section{Carolina Sotelo}

Universidad El Bosque

\section{Juan Esteban Garcia-Robledo}

Mayo Clinic Scottsdale: Mayo Clinic Arizona

\section{Nicolas Santoyo}

Universidad El Bosque

\section{Christian Rolfo}

Icahn School of Medicine at Mount Sinai Tisch Cancer Institute

\section{Rafael Rosell}

Catalan Institute of Oncology: Institut Catala d' Oncologia

\section{Oscar Arrieta}

Mexico National Cancer Institute: Instituto Nacional de Cancerologia

\section{Research Article}

Keywords: glioblastoma, EGFR, amplification, mutation, tyrosine kinase inhibitor, bevacizumab, osimertinib

Posted Date: July 27th, 2021

DOI: https://doi.org/10.21203/rs.3.rs-698468/v1

License: (a) (i) This work is licensed under a Creative Commons Attribution 4.0 International License. Read Full License 


\section{Abstract}

Background: Amplification of EGFR and its active mutant EGFRvIII are common in glioblastoma (GB). While EGFR and EGFRVIII play critical roles in pathogenesis, targeted therapy with EGFR-tyrosine kinase inhibitors (TKIs) or antibodies has shown limited efficacy. To improve the likelihood of effectiveness, we targeted adult patients with recurrent GB enriched for simultaneous EGFR amplification and EGFRVIIImutation, with osimertinib/bevacizumab at doses described for non-small cell lung cancer (NSCLC).

Methods: We retrospectively explored whether previously described EGFRVII/mutation in association with EGFR gene amplification could predict response to osimertinib/bevacizumab combination in a subset of 15 patients treated at recurrence. The resistance pattern in a subgroup of subjects is described using a commercial NGS panel in liquid biopsy.

Results: There were ten males (66.7\%), and the median patient's age was 56 years (range 38-70 years). After their initial diagnosis, 12 patients underwent partial $(26.7 \%)$ or total resection (53.3\%). Subsequently, all cases received IMRT and concurrent and adjuvant temozolomide (TMZ; the median number of cycles 9, range 6-12). The median follow-up after recurrence was 17.1 months $(95 \% \mathrm{Cl} 12.3-22.6)$. All patients received osimertinib/bevacizumab as a second-line intervention with a median progression-free survival (PFS) of 5.1 months (95\% $\mathrm{Cl} 2.8-7.3$ ) and overall survival (OS) of 9.0 months (95\% $\mathrm{Cl} 3.9-14.0)$. The PFS6 was $46.7 \%$, and the overall response rate (ORR) was $13.3 \%$. After exposure to the osimertinib/bevacizumab combination, the main secondary alterations were MET amplification, STAT3, IGF1R, PTEN, and PDGFR.

Conclusions: While the osimertinib/bevacizumab combination was marginally effective in most GB patients with simultaneous EGFR amplification plus EGFRvIII mutation, a subgroup experienced a long-lasting meaningful benefit. The findings of this brief cohort justify the continuation of the research in a clinical trial. The pattern of resistance after exposure to osimertinib/bevacizumab includes known mechanisms in the regulation of $E G F R$, findings that contribute to the understanding and targeting in a stepwise rational this pathway.

\section{Introduction}

Glioblastoma (GB) or grade IV glioma according to the 2016 WHO classification of brain malignancies, is the most common primary malignant tumor of the central nervous system (CNS), with an estimated incidence of $10 / 100.000$ population, occurring frequently in people aged between $55-60$ years $(1,2)$. The prognosis of GB remains poor despite first-line therapy, and the median overall survival (OS) is 12-15 months (2), while the 5year survival does not exceed $10 \%$ of patients(3). After first line therapy, GB almost always recurs with poorer prognosis (median PFS of 1.5-6 months and median OS of 2-9 months) (4-6), especially due to intra-tumor heterogeneity, on cellular and molecular levels (7). Once GB progresses after first-line therapy, treatment options are limited, and recurrent GB (rGB) medical care remains a challenge. Loco-regional therapy (surgery and reirradiation) may be evaluated in selected cases while traditional systemic therapy has shown limited efficacy. In recent years, with greater knowledge of the underlying molecular features, a multitude of new drugs and combination regimens have been tested for efficacy in rGB patients (8-10).

Currently, astrocytic gliomas with a wild-type IDH and histone $\mathrm{H} 3$ status, necrosis and/or microvascular proliferation are classified as IDH-wild-type, WHO grade 4 glioblastomas $(1,11)$. In the absence of necrosis or 
microvascular proliferation, such tumors should be evaluated for glioblastoma-associated EGFR gene amplification, TERT promoter mutations and/or the $+7 /-10$ signature $(11,12)$. If one of these alterations is detected, they are classified as IDH-wild-type glioblastomas given their association with a poor prognosis, even in the absence of necrosis and microvascular proliferation $(1,13)$. MGMT promoter methylation has limited diagnostic value for rGB but can guide therapy, whether to choose chemotherapy with alkylating or other agents in IDH-wild-type gliomas (2). Next-generation sequencing (NGS) based gene panels could enable the assessment of all or most genetic and chromosomal aberrations relevant for diagnosis using a single assay (14, 15). Furthermore, array-based DNA methylation profiling and RNAseq has emerged as a powerful novel diagnostic method that is independent of histology and useful in the routine diagnostic and therapeutic work-up $(16,17)$.

Epidermal growth factor receptor (EGFR) is a transmembrane receptor tyrosine kinase of the ERBB family. Upon binding to ligands which include epidermal growth factor (EGF), Transforming growth factor alpha (TGF-a), amphiregulin, betacellulin, heparin-binding EGF (HB-EGF), epigen or epiregulin, EGFR forms homodimers or heterodimers with other ERBB family members (18). Dimerization of EGFR leads to transphosphorylation (autophosphorylation) of its C-terminal tail, which serves as the docking site for SRC homology 2 (SH2) domaincontaining signaling proteins including growth factor receptor-bound protein 2 (GRB2), phosphoinositide 3kinase (PI3K), SRC homology 2 domain-containing transforming protein 1 (SHC1) and signal transducer and activator of transcription (STAT) proteins (18-20). These signaling proteins regulate downstream physiological and pathological processes. The EGFR pathway represents a particularly attractive therapeutic target in GB because is dysregulated in most human malignant gliomas through overexpression, amplification, and activating mutations.

Amplification of the EGFR gene occurs in $\approx 55 \%$ of IDH-wild-type GB patients and is associated with high levels of EGFR protein $(21,22)$. In contrast, a separate group of EGFR deletions and point mutations is found frequently in GB. EGFR deletions in GB include EGFRVI (N-terminal deletion), vII(deletion of exons 14-15), vIII (deletion of exons 2-7), $v I V$ (deletion of exons 25-27), $v V$ (deletion of exons 25-28), among which $v / l$ and $v / I I$ are oncogenic (23). In addition, point mutations in the extracellular region of EGFR (R108K, A289V/D/T, G598D among others) keep the receptor in an active state and are identified in $24 \%$ of $\mathrm{GB}$ cases $(22,23)$. Among $E G F R$ mutants found in GB, the most common is EGFRVIII, occurring after amplification of EGFR and is felt to represent a late event $(24,25)$.

Several classes of EGFR inhibitors have been developed, including small-molecule tyrosine kinase inhibitors (TKIs), antibodies, immunotoxin conjugates, and antisense oligonucleotides. Because intracranial distribution of many agents is limited, the use of small-molecule TKIs offers a theoretical advantage (26). However, several early trials in unselected patients with GB have reported limited efficacy with EGFR-selective TKIs. Reasons why this lack of efficacy include limited brain penetration of drugs (first-generation TKIs do not cross the BBB -BloodBrain Barrier - effectively), complex tumor heterogeneity (there are at least four characterized major molecular forms of EGFR), redundant signaling pathways, and limited potency in first and second-generation drugs because they aim targets that proved to be different in GB than other type of EGFR-amplified tumors (27-29). Osimertinib, a third-generation EGFR-TKI that effectively penetrates the BBB, was recently found to be $>10$ times more efficient than the first-generation EGFR inhibitors in inhibiting GB proliferation, inducing cell cycle arrest which significantly inhibited colony formation, migration, and invasion through downregulation of EGFR/ERK signaling (30). Furthermore, osimertinib inhibits the constitutive activity of EGFRVIII with high potency (< 100 
$\mathrm{nM}$ ) in a tyrosine kinase-dependent manner (31). In addition, it has been recently found that cells expressing mutant EGFRVIII are prone to starvation and hypoxia-induced cell death (32). This research supports the idea that using anti-VEGF agents that produce a low-oxygen environment could promote EGFRVII-mutant hypoxiainduced cell death. Albeit some initial equivocal results, there is sufficient biological evidence to consider that patients with EGFR-amplified and EGFRVIII-mutant GB could benefit from combined therapy with osimertinib and bevacizumab.

\section{Methods}

We conducted a retrospective review of rGB patients with simultaneous EGFR amplification and EGFRVIII mutation, that were treated with a fixed dose of osimertinib $(80 \mathrm{mg} / \mathrm{d})$ and bevacizumab $(15 \mathrm{mg} / \mathrm{kg}$, every three weeks) from March 2016 through September 2020 at Clínica del Country (Bogotá, Colombia). The endpoints were progression-free survival (PFS) at six months (PFS6), overall response rate (ORR), overall survival (OS) and safety. Two pairs of locally certified neuroradiologists evaluated responses using RANO criteria (33). Concerns about the response to radiation therapy or changes caused by it were settled via consensus among the observers. During treatment, all patients were treated according to best international practices.

Baseline demographic and clinical data for each patient were collected by individual chart review from the institution's electronic medical record system. Charts were scrutinized for treatment dose adjustments, discontinuations, and toxicities as defined by the Common Terminology Criteria for Adverse Events (CTCAE v5) (https://ctep.cancer.gov/protocoldevelopment/electronic_applications/docs/ctcae_v5_quick_reference_5x7.pdf). In general, both drugs were administered until disease progression or intolerance occurred. When significant toxic effects were discovered, osimertinib treatment was halted and resumption was set at $40 \mathrm{mg}$ per day. When there was significant toxicity, bevacizumab was discontinued but, whenever possible, resumed at the same dose. If patients experienced severe adverse effects (gastrointestinal perforation [any grade], thromboembolism, pulmonary hemorrhage [grade 2] or other hemorrhages, allergic reaction, or cardiac toxic effects [grade 3]), bevacizumab treatment was discontinued. Bevacizumab was also halted if patients did not recover from an adverse effect that required suspension within 42 days (i.e., serum creatinine $>1.5 \mathrm{mg} / \mathrm{dL}$, proteinuria greater than $2+$, or hypertension [grade 4]).

This study was conducted in compliance with the Declaration of Helsinki (34) and with the International Conference on Harmonization Good Clinical Practice Guidelines. The protocol was approved by an institutional review board (ONCOLGroup Platform - Registration No. 2018/21904, Cayre, Clínica del Country, Bogotá, Colombia). All included patients provided signed informed consent and accepted the tumor tissue analysis. An Institutional Review Board and Privacy Board waiver was obtained to facilitate retrospective collection of clinical, pathologic and molecular data.

\section{Genomic profiling}

In all cases, the genomic profiling was done using validated methods. Studies were conducted using an NGSbased assay (Foundation-One ${ }^{\circledR}$ ) performed in a laboratory accredited by CLIA and The College of American Pathologists (Foundation Medicine, Cambridge, MA, http://www.foundationmedicine.com). An expert pathologist (PA) reviewed hematoxylin and eosin (H\&E) stained slides to confirm the GB diagnosis and ensure adequate formalin-fixed, paraffin-embedded (FFPE) specimen quality. Samples were considered to have 
acceptable quality if: sample volume $>1 \mathrm{~mm}^{3}$, nucleated cellularity $>80 \%$ or $>30,000$ cells, and $>20 \%$ tumor nuclei. When required, microdissection was performed to enrich samples with $<20 \%$ tumor nuclei. In brief, $50 \mathrm{ng}$ of DNA was extracted from formalin-fixed, paraffin-embedded tissue blocks of tumor samples. Hybridization capture of 3,320 exons from up to 315 cancer-related genes and select introns of 28 genes commonly rearranged in cancer were applied to the extracted DNA. The samples were sequenced to high, uniform coverage, with a mean sequencing depth of 5643 , as previously described (35). GAs, including base substitutions, small insertions or deletions (indels), rearrangements, and copy number alterations, were determined and reported for these samples. MGMT (0-6-Methylguanine-DNA Methyltransferase) promoter methylation status was obtained from diagnostic tumor specimens by methylation-specific PCR bisulfite-treated DNA as previously described (36).

The commutations found at diagnosis were recorded in all patients. In 8 cases, an F-One liquid test was performed at the time of progression to osimertinib/bevacizumab; 5 of them (62\%) had positive results with novel alterations described in the results.

\section{Statistical design and analysis}

Patient characteristics were summarized using median, interquartile range, and minimum/maximum values for continuous variables and frequency (\%) for categorical variables. PFS was defined as the time interval between the date of first osimertinib/bevacizumab dose and the date of disease progression or death from any cause, whichever occurred first. Patients who were alive without disease progression at the time of the last follow-up were censored. OS was defined as the time interval between the first Osimertinib plus bevacizumab dose and the date of death due to any cause. Patients that were alive were censored at the last follow-up. The PFS, PFS6, and OS with 95\% Cl were estimated using Kaplan-Meier curves. A Cox regression model was used to estimate the adjusted HR stratified by response to prior chemoradiotherapy. The difference in the ORR was assessed using the $\chi^{2}$ test. Statistical analyses were conducted with SPSS 26.0 version (Statistical Package for the Social Sciences, IBM, Armonk, NY, US).

\section{Results}

From March 2016 through September 2020, 61 patients with GB who harbored EGFR gene amplification were diagnosed in two reference centers for the treatment of brain tumors in Bogotá, Colombia. Like previous reports (37), EGFRvII/ was detected in 40\% ( $\mathrm{N}=24)$ of this EGFR-amplified GB cohort. EGFR amplification and mutation status were determined from the initial diagnostic tumor specimen in 47 (77\%) patients and from recurrent, immediate pretreatment tumor tissue in 14 (23\%) cases (Fig. 1). All tumors were IDH1/2 wild type, consistent with previously described EGFR amplification and IDH mutation inverse correlation $(38,39)$. Seven patients $(46.7 \%)$ had MGMT promoter methylation and the mean number of commutations found in the tumor tissue at the time of diagnosis was 3 (SD +/-1) (Supplementary Table 1). Table 1 includes the baseline patient demographics and disease characteristics.

Table 1. Baseline patient demographics and disease characteristics. 


\begin{tabular}{|c|c|}
\hline Variable & $N=15(\%)$ \\
\hline \multicolumn{2}{|l|}{ Age } \\
\hline$\geq 65$ years & $11(73.3)$ \\
\hline$₫ 64$ years & $4(26.7)$ \\
\hline \multicolumn{2}{|l|}{ Sex } \\
\hline Male & $10(66.7)$ \\
\hline Female & $5(33.3)$ \\
\hline \multicolumn{2}{|l|}{ Karnofsky index } \\
\hline$<80 \%$ & $1(13.3)$ \\
\hline$>80 \%$ & $13(86.7)$ \\
\hline \multicolumn{2}{|l|}{ RPA scale } \\
\hline 5 & $14(93.3)$ \\
\hline 6 & $1(6.7)$ \\
\hline \multicolumn{2}{|c|}{ Compromise of eloquent areas } \\
\hline Yes & $5(33.3)$ \\
\hline No & $11(66.7)$ \\
\hline \multicolumn{2}{|c|}{ Corpus callosum infiltration } \\
\hline Yes & $2(13.3)$ \\
\hline No & $13(86.7)$ \\
\hline \multicolumn{2}{|l|}{ Tumor location } \\
\hline Frontal lobe & $4(26.7)$ \\
\hline Temporal lobe & $8(53.3)$ \\
\hline Parietal lobe & $2(13.3)$ \\
\hline$>2$ lobes & $1(6.7)$ \\
\hline \multicolumn{2}{|c|}{ Baseline neurological status } \\
\hline Altered & $4(26.7)$ \\
\hline Normal & $11(73.3)$ \\
\hline \multicolumn{2}{|l|}{ Tumor diameter } \\
\hline$<3 \mathrm{~cm}$ & $6(40,0)$ \\
\hline$>3 \mathrm{~cm}$ & $8(53.3)$ \\
\hline ND & $1(6.7)$ \\
\hline
\end{tabular}




$\begin{array}{ll}\text { Biopsy/subtotal } & 7(46.7) \\ \text { Total } & 8(53.3)\end{array}$

$\begin{array}{ll}\text { Pseudoprogression } & \\ \text { Yes } & 4(26.7) \\ \text { No } & 11(73.3)\end{array}$

There were 10 males (66.7\%), and the median patient's age was 56 years (range 38-70 years). Following their initial diagnosis, 4 patients underwent partial $(\mathrm{N}=4 / 26.7 \%)$ and 8 total resections $(\mathrm{N}=8 / 53.3 \%)$. In three cases $(20 \%)$ it was only possible to perform a biopsy due to the involvement of some eloquent area. Subsequently, all cases received IMRT, with 14 (93\%) completing 60 Gy and concurrent and adjuvant temozolomide (TMZ; median number of cycles 9 , range $6-12)$. In addition, four patients had completed 12 cycles of TMZ. The overall response rate (ORR) to first-line therapy was $40 \%(N=6)$, with $40 \%$ achieving stable disease $(N=6)$, and three patients progressing $(20 \%)$. Four patients $(26.7 \%)$ presented pseudoprogression; in two cases, it was symptomatic and required steroid support and subsequent tapering. This finding was only seen in the subset of patients who had a partial response. The PFS for the first-line treatment was 9.7 months ( $95 \% \mathrm{Cl} 6.5-12.8)$ (Supplementary Fig. 1), and the median OS from diagnosis was 17.0 months ( $95 \% \mathrm{Cl} 12.8-21.1)$ (Supplementary Fig. 2). Following progression, six patients underwent resection of recurrent tumor, with five achieving optimal surgery (83\%). In addition, three of them were treated with re-radiation using fractionated stereotaxic radiosurgery. OS by MGMT favored the methylated population [MGMT methylated 26.9 months $(95 \%$ $\mathrm{Cl} 25.1-28.6)$ vs. Unmethylated MGMT 14.1 months $(95 \% \mathrm{Cl} 12.1-16.0 ; \mathrm{P}=0.001)]$, finding that was reproduced for the PFS achieved with chemoradiation followed by temozolomide [MGMT methylated 15.1 months $(95 \% \mathrm{Cl}$ 13.2-16.9) vs. Unmethylated MGMT 7.6 months (95\% $\mathrm{Cl} 6.9-8.3 ; \mathrm{P}=0.001)]$.

\section{Outcomes for osimertinib/bevacizumab combination}

Fifteen patients who had simultaneous EGFR amplification and EGFRvII/variant received osimertinib plus bevacizumab as a second-line intervention. At the time of the study report, $80 \%(\mathrm{~N}=12)$ of the patients had died, and the rest were in follow-up receiving active treatment. The median follow-up was 17.1 months $(95 \% \mathrm{Cl} 12.3-$ 22.6) and all had a Karnofsky index greater than $70 \%$ at the time of starting treatment. Only four patients had a neurological deficit considered mild to moderate. Seven (46.7\%) of 15 patients achieved PFS6, the median PFS was 5.1 months (95\% Cl 2.8-7.3) (Fig. 2A) and the median OS from osimertinib/bevacizumab was 9.0 months (95\% Cl 3.9-14.0) (Fig. 2B). The median number of bevacizumab cycles was 7.0 (range, 3 to 22), one patient (6.7\%) achieved a complete response, another had a partial response (6.7\%), and transient stable disease was observed in 10 cases. The ORR was $13.3 \%$ and the clinical benefit was $80 \%$. Figure 3 shows a representative case that achieved a good response and PFS after osimertinib/bevacizumab. In a subset of eight patients, a secondary molecular study was performed by NGS in liquid biopsy; five had positive results with various alterations found after disease progression to the osimertinib/bevacizumab combination. Only two cases preserved the EGFR amplification and one the EGFRVIII alteration. MET amplification was documented in two cases, and in one that lost both baseline EGFR and CDKN2A/B alterations, a secondary EGFR A289V (Mutant Allele Frequency Percentage - MAF\%; MAF 6.1\%) point mutation plus an alteration in STAT3 T433I (MAF 1.7\%) were found. Other genes related to osimertinib/bevacizumab resistance were IGF1R (MAF 0.56\%), PTEN, and 
PDGFR (MAF 1.6\%). Figure 4 illustrates genomic findings in five selected patients comparing initial tissue and blood analysis after progression. Two of the patients with higher PFS (15.5 and 9.1 months) after the start of osimertinib/bevacizumab developed MET amplification, opening the possibility of considering the use of specific MET inhibitors as rescue therapy. MGMT promoter methylation also positively affected PFS for recurrent disease treated with osimertinib/bevacizumab [MGMT methylated 8.3 months (95\% $\mathrm{Cl}$ 4.7-11.8) vs. Unmethylated MGMT 4.1 months (95\% Cl 1.6-6.5; P = 0.02)].

Eleven patients (73.3\%) received a third-line treatment, 9 were exposed to BCNU/ bevacizumab and 2 to nivolumab/bevacizumab. Seven patients achieved stable disease with a PFS of 4.1 months (95\% $\mathrm{Cl} 3.6-4.5)$. The best results with the third line were patients that achieved PFS6 with osimertinib/bevacizumab.

\section{Safety and tolerability}

Most treatment-related adverse events (AEs) were controllable with standard care, and the overall toxicity profile was consistent with previous reports in multiple solid tumors (Table 2). Common grade $\geq 2$ AEs considered at least possibly related to osimertinib and bevacizumab combination included hypertension (40\%), rash (33.3\%), fatigue (33.3\%), diarrhea (26.8\%), paronychia (20.1\%), and mild proteinuria (20.1\%). Grade $\geq 3$ related AEs included diarrhea (13.3\%), fatigue (13.3\%), hypertension $(6.7 \%)$ and paronychia $(6.7 \%)$. There was no severe bleeding or embolism, and one patient experienced transient interstitial grade 1 lung disease. Four patients (26.4\%) experienced temporary dose interruption with osimertinib in one of the cases for 16 days due to grade 2-3 diarrhea. After correcting the event, he restarted with $40 \mathrm{mg}$ with occasional stools. No patient required temporary or permanent suspension of bevacizumab. 
Table 2

Toxicity profile of osimertinib/bevacizumab combination as a second line therapy in rGB.

\begin{tabular}{|llll|}
\hline Toxicicty & N=15(\%) & \\
\hline Toxicity grade & All & Grade 1-2 & Grade 3 \\
\hline Hypertension & $6(40)$ & $5(33.3)$ & $1(6,7)$ \\
\hline Rash & $5(33.3)$ & $5(33.3)$ & - \\
\hline Fatigue & $5(33.3)$ & $3(20.0)$ & $2(13.3)$ \\
\hline Diarrhea & $4(26.8)$ & $2(13.3)$ & $2(13.3)$ \\
\hline Proteinuria & $3(20.1)$ & $3(20.1)$ & - \\
\hline Thrombocytopenia & $1(6.7)$ & $1(6.7)$ & - \\
\hline Decreased albumin & $2(13.3)$ & $2(13.3)$ & - \\
\hline Neutrophil decreased & $1(6.7)$ & $1(6.7)$ & - \\
\hline Paronychia & $3(20.1)$ & $2(13.3)$ & $1(6.7)$ \\
\hline Increased aspartate aminotransferase & $2(13.3)$ & $2(13.3)$ & - \\
\hline WBC decreased & $2(13.3)$ & $2(13.3)$ & - \\
\hline Creatinine increased & $1(6.7)$ & $1(6.7)$ & - \\
\hline Increased alkaline phosphatase & $1(6.7)$ & $1(6.7)$ & - \\
\hline Interstitial lung disease & $1(6.7)$ & $1(6.7)$ & \\
\hline
\end{tabular}

\section{Discussion}

This study is a brief series of patients with EGFR-amplified and EGFRvII-mutant GB that received combined therapy with osimertinib / bevacizumab for recurrent GB. This targeted intervention achieved a PFS6 of $46.7 \%$, a higher outcome than lomustine or bevacizumab monotherapy, where the PFS6 ranged between 13\% and 16\% (40). Despite the sampling limitation, the PFS6 obtained with osimertinib/bevacizumab in this selected population was similar to that achieved with the lomustine/bevacizumab regimen in the BELOB trial (40). In addition, OS with osimertinib/bevacizumab was equal to that achieved in the control arm (9.0 months) of study EF-14, superior to regorafenib (7.4 months) (41), and slightly lower than that obtained with the tumor-treating fields (TTFields; 11.8 months) (42).

Many correlative analyses of EGFR status in clinical trials for glioblastoma found it to be prognostically significant, though a larger meta-analysis failed to confirm this. (43). In all large genome-wide cancer studies, it became a key molecule for glioma, which has favored the exploration of multiple molecules even without much success. Osimertinib is a 3rd generation irreversible EGFR tyrosine kinase inhibitor, targeting the ATP-binding pocket of the kinase domain by formation of a covalent bond with Cys 797. Additionally, osimertinib has been shown to have efficacy in brain metastases, conveying the ability to cross the blood brain barrier in clinically relevant concentrations superior to that of other inhibitors (in naïve patients with advanced EGFR-mutated Non- 
small cell lung cancer and CNS metastases, the pooled ORR and DCR of osimertinib were $71 \%$ and $93 \%$, respectively, and the combined median PFS was 12.21 months) (44). However, osimertinib has relatively low affinity for the kinase domain of both wild-type EGFR and EGFRvIII, as it was specifically designed to target the mutant T790M kinase domain (IC50 $1 \mathrm{nM}$ ) and spare the wild-type variant (IC50 $184 \mathrm{nM}$ ) (45). So far, no human trials with osimertinib in selected GB patients have been completed to date. There are some reports in the literature of efficacy, including one patient with EGFR A289V and C628F point mutations and EGFR copy number gain that received osimertinib at multifocal recurrence, with near-complete response in one lesion but discordant simultaneous progression at another site. The progressing lesion acquired an EGFRVIIImutation and continued to exhibit EGFR copy number gain (46).

Previously, Lassman et al. evaluated EGFR expression/signaling on gefitinib and erlotinib using Western blot (anti-pEGFR Tyr 1068 antibodies) on GB datasets from NABTC 01-03 and 00-01 (47). Erlotinib and gefitinib treatment did not consistently affect EGFR activity pre-/post- erlotinib or gefitinib, whether by pEGFR, pERK, or pAKT. This suggested that erlotinib and gefitinib did not effectively inhibit EGFR phosphorylation or signaling in these tumors. Lack of effect on downstream signal transduction despite apparent appropriate inhibition appears to be mediated through alternative escape pathways, such as MET, IGF1R (insulin growth factor receptor 1), and PI3K (47).

In our study, the post-progression analysis of the liquid biopsy (performance of post-progression liquid biopsy $62 \%$ ) found alterations in IGFR1R, PTEN, AKT3, PDGFR, STAT3, and MET amplification, the latter associated with the best response after exposure to osimertinib/bevacizumab. In the same way, Mellinghoff et al. treated 49 recurrent glioblastoma patients with erlotinib (at doses from 150 to $500 \mathrm{mg}$ ) and gefitinib (from 150 to 1000 $\mathrm{mg}$ ) and found that PTEN loss was associated with resistance to EGFR tyrosine kinase inhibitors (48). There is also substantial preclinical evidence that activation of the PI3K, MET, or the IGF1R pathways confers resistance in a similar manner to what occurs with first line osimertinib in patients with NSCLC and actionable mutations in EGFR (49-52).

There is increasing experimental and clinical evidence that activation of EGFR and downstream signaling pathways render specific subgroups of GBs vulnerable to hypoxia-inducing therapies. Hypoxia-induced cell death in GB models is preceded by ATP depletion, increase in glycolysis and higher levels of mitochondrial superoxides which have been associated with decreased metabolic flux and decreased NADPH/NADP + ratio (53). The difficulty in effectively tackling tumor cells lies on the ample heterogeneity amongst various GB samples and models. There are different point mutations in the extracellular region of EGFR whose exact biological implications still elude us. A plethora of cell signaling pathways mediated by EGFR can be divided in those originating from plasma membrane: RAS/mitogen activated protein kinase/extracellular signal-regulated kinase (RAS/MAPK/ERK) pathway, PI3K/protein kinase B (PKB/AKT) pathway, Janus kinase/STAT (JAK/STAT) pathway and protein kinase $C$ (PKC) pathway (4). The second group includes non-plasma membrane signaling pathways: nuclear (DNA-PK, PCNA, histone H4 and STAT proteins) and mitochondrial (COXII) (4).

Interestingly, interaction of JAK2 with EGFR within the JAK/STAT pathway has shown to confer resistance to EGFR inhibitors and when EGFRVIII is expressed, STAT3 forms a complex with EGFRVIII that drives malignant transformation and initiates mesenchymal transformation in high-grade gliomas (54-56). Active EGFR recruits and activates phospholipase C (PLC) which in turn activates PKC. PKCs downstream effectors include p53 and p21, cell growth regulators (RAS-RAF1 and others that may be tumor suppressants or oncogenes depending on 
context). In GB, PKC isoenzymes show pro-tumorigenic functions. High levels of isoform PLC-gamma are correlated with survival and inhibition of PKC proved decreased viability of GB cells; hence PKC is a critical signaling target (57-59). EGFR can phosphorylate EGFRvIIIto activate STAT3/5 in the nucleus and facilitate tumour progression. This could be one of many biological correlates that explains why patients with EGFRvIII have shortened survival, strictly dependent on its kinase activity. EGFRVIII phosphorylates SFKs which promotes mitochondrial localization of EGFRvIII and increases cell survival under low glucose conditions (60). EGFRvIII also activates c-SRC that promotes angiogenesis via VEGF secretion and growth of cells expressing wild-type EGFR via paracrine interleukin 6 (IL-6). These preclinical findings favor the use of osimertinib / bevacizumab, and in our case the presence of acquired mutations in STAT3 and AKT confirm the escape through these pathways (60). Figure 5 illustrates the EGFRvIII related pathways and angiogenesis in GB cells (see Supplementary data to Fig. 5 for a detailed explanation).

Recently, Struve et al. reported that EGFRvIll expression is associated with prolonged survival, but only in patients with MGMT promoter methylation (61). In addition, using isogenic cell lines with high EGFRvIII expression they demonstrate that EGFRVIII increases TMZ sensitivity through DNA double-strand breaks and a pronounced S/G2-phase arrest. As well, they demonstrated a greater expression of DNA mismatch repair (MMR) proteins in EGFRvIII positive cells and patient tumor samples, which was most pronounced for MSH2 and MSH6. EGFRvIIIspecific knockdown reduced MMR protein expression, thereby increasing TMZ resistance (61). In opposition, Chi et al. found that the MGMT promoter methylation was not associated with clinical response to dacomitinib in a cohort of GB patients with EGFR amplification and the EGFRv/l/mutation (28). In our study, MGMT promoter methylation significantly impacted OS $(P=0.001)$ (Supplementary Fig. 3 ) as well as PFS with initial treatment (chemoradiation therapy followed by temozolomide; $P=0.001$ ) (Supplementary Fig. 4), and PFS with the osimertinib / bevacizumab combination $(P=0.02)$ (Supplementary Fig. 5), favoring the hypothesis that relates the overexpression of the EGFRVIII mutation with the response to the alkylating agent and the direct inhibition of EGFR.

Eskilsson et al. favored our hypothesis by finding a slight correlation between EGFR and VEGFA expression when considering only GB samples with EGFR amplification ( $P=0.028)$, but not in those without EGFR amplification. Interestingly, strong correlation between EGFR and VEGFA expression in GB samples with EGFR amplification was limited to those that additionally harbored EGFRVIII $(P=0.031)$ and/or EGFR missense mutations $(P=0.037)(62)$. In addition, Chagoya et al. tested the efficacy of osimertinib in vivo and in vitro finding that EGFRVIII GBs are heterogeneous in terms of expression of EGFRvIII, by the extent of EGFRVIIIs tyrosine kinase activity, and in the expression of several other key genes (31). To our knowledge, previous clinical trials did not consider the heterogeneity of EGFR positive GBs. For example, the failed EGFRvIII-vaccine trial that included all EGFRvIII positive GB patients (63) or the recent fall of depatuxizumab in the INTELANCE-1 study (64). Part of the answer may lie in the recent findings of the REGOMA study, where a molecular signature associated with prolonged survival in GB patients treated with regorafenib was found (65). Santangelo et al. reported elevated expression levels of HIF1A mRNA and CDKN1A mRNA and reduced expression levels of miR93-5p, miR-3607-3p, and miR-301a-3p in tumor tissue at first surgery, can identify a subgroup of patients treated with regorafenib with favorable benefit. The selection of patients with EGFR amplification who have the EGFRvIII mutation and high levels of VEGF or HIF1 may be the best candidates to consider the use of the osimertinib/bevacizumab combination. 
In conclusion, our findings are intriguing and support the idea of better understanding all signaling pathways implicated in EGFR-positive GB patients. To the best of our knowledge, this is the first report of a group of patients with EGFR amplification and EGFRVIIImutations who were treated with osimertinib/bevacizumab. Some had a positive impact on PFS and PFS6, which validates the transition to a confirmatory clinical trial. Even so, more preclinical studies are needed to determine the molecular signatures of EGFR GBs that may make these tumors sensitive to the combination.

\section{Declarations}

\section{Acknowledgments}

The authors are grateful for the generous contribution from the Silberman family and Francisco Castillo (Fundación Neuronas con Corazón) for altruistically promoting the development of brain tumor research in Colombia.

\section{Ethical approval}

This study was reviewed and approved by ONCOLGroup Platform - Registration No. 2018/21904, Cayre, Clínica del Country, Bogotá, Colombia. All included patients provided signed informed consent and accepted the tumor tissue analysis. An Institutional Review Board and Privacy Board waiver was obtained to facilitate retrospective collection of clinical, pathologic and molecular data.

\section{Data availablity statement}

The datasets presented in this article are not readily available because of the Colombian organic law of data protection that limits access to genetic information in an open format. Requests to access the datasets should be directed to the corresponding author, who will release it upon formal request to the Ministry of Health of Colombia following the requirements of Law 1581 of 2012, paragraph 201811601170851 of 2018.

\section{Funding}

Supported by Foundation for Clinical and Applied Cancer Research- FICMAC (Bogotá, Colombia) research grant 014-2018.

\section{Author Contributions}

AFC, OA, ARP, EJ, FH, DG, JFR, HC and JAM planned and coordinated the study. DJV, CP, FS, CO, AM, SB, NU, DP, $L R, Z L Z, E J$ and $C S$ reviewed patient records and composed the database. LR reviewed all histopathology studies. JR, JA, JGR, NS and ARP performed DNA extraction and library preparation and MGMT methylation analysis. AFC, ARP, OA, CR, and RR performed all statistical analysis and data interpretation. AFC, ARP, OA and DJV wrote the initial draft of the manuscript. All authors contributed to the article and approved the submitted version.

\section{Conflict of interest}


Andrés F. Cardona discloses financial research support from Merck Sharp \& Dohme, Boehringer Ingelheim, Roche, Bristol-Myers Squibb, Foundation Medicine, Roche Diagnostics, Termo Fisher, Broad Institute, Amgen, Flatiron Health, Teva Pharma, Rochem Biocare, Bayer, INQBox and The Foundation for Clinical and Applied Cancer Research - FICMAC. Additionally, he was linked and received honoraria as an advisor, participate in speakers' bureau. He gave expert testimony to EISAI, Merck Serono, Jannsen Pharmaceutical, Merck Sharp \& Dohme, Boehringer Ingelheim, Roche, Bristol-Myers Squibb, Pfizer, Novartis, Celldex Therapeutics, Foundation Medicine, Eli Lilly, Guardant Health, Illumina, and Foundation for Clinical and Applied Cancer Research FICMAC.

Oscar Arrieta reports personal fees from Pfizer, grants and individual fees from Astra Zeneca, grants and individual fees from Boehringer-Ingelheim, Lilly, Merck, Bristol Myers Squibb, Roche, outside the submitted work.

Christian Rolfo reports relation with Mylan, Archer Biosciences, Oncopass, Inivata, Merck Serono Novartis, MSD, Boehringer Ingelheim, Guardant Health, etc AstraZeneca as part of Speakers' Bureau. Also, he received research funding from Pfizer and had uncompensated Relationships with OncoDNA, Biomark, and Guardant Health.

Leonardo Rojas received honoraria as an advisor, participate in speakers' bureau from Merck Sharp \& Dohme, Boehringer Ingelheim, Roche, Bristol-Myers Squibb, Astra Zeneca and Eli Lilly. Additionally, he was linked and received honoraria as researcher.

The other authors have no conflicts to declare.

\section{References}

1. Louis DN, Perry A, Reifenberger G et al (2016) The 2016 World Health Organization Classification of Tumors of the Central Nervous System: a summary. Acta Neuropathol 131:803-820

2. Weller $M$, van den Bent $M$, Preusser $M$ et al (2020) EANO guidelines on the diagnosis and treatment of diffuse gliomas of adulthood. Nat Rev Clin Oncol 18(3):170-186. doi:10.1038/s41571-020-00447-z

3. McNeill KA (2016) Epidemiology of Brain Tumors. Neurol Clin 34(4):981-998. doi:10.1016/j.ncl.2016.06.014

4. Chen W, Wang Y, Zhao B et al (2021 Mar) Optimal Therapies for Recurrent Glioblastoma: A Bayesian Network Meta-Analysis. Front Oncol 29:11:641878. doi:10.3389/fonc.2021.641878

5. McBain C, Lawrie TA, Rogozińska E et al. Treatment options for progression or recurrence of glioblastoma: a network meta-analysis. Cochrane Database of Systematic Reviews 2021, Issue 1. Art. No.: CD013579. DOI: 10.1002/14651858.CD013579.pub2

6. Birzu C, French P, Caccese M et al (2020) Recurrent Glioblastoma: From Molecular Landscape to New Treatment Perspectives. Cancers (Basel). Dec 26;13(1):47. doi: 10.3390/cancers13010047

7. Vartanian A, Singh SK, Agnihotri S et al (2014 Sep) GBM's multifaceted landscape: highlighting regional and microenvironmental heterogeneity. Neuro Oncol 16(9):1167-1175. doi:10.1093/neuonc/nou035. Epub 2014 Mar 18

8. Zhao YH, Wang ZF, Pan ZY et al. A Meta-Analysis of Survival Outcomes Following Reoperation in Recurrent Glioblastoma: Time to Consider the Timing of Reoperation. Front Neurol. 2019 Mar 26;10:286. doi:

10.3389/fneur.2019.00286

Page 14/22 
9. Wang $Y$, Xing D, Zhao $M$ et al. The Role of a Single Angiogenesis Inhibitor in the Treatment of Recurrent Glioblastoma Multiforme: A Meta-Analysis and Systematic Review. PLoS One. 2016 Mar 23;11(3):e0152170. doi: 10.1371/journal.pone.0152170

10. Kazmi F, Soon YY, Leong YH et al (2019 Mar) Re-irradiation for recurrent glioblastoma (GBM): a systematic review and meta-analysis. J Neurooncol 142(1):79-90. doi:10.1007/s11060-018-03064-0. Epub 2018 Dec 6

11. Brat DJ, Aldape K, Colman H et al (2018 Nov) cIMPACT-NOW update 3: recommended diagnostic criteria for "Diffuse astrocytic glioma, IDH-wildtype, with molecular features of glioblastoma, WHO grade IV". Acta Neuropathol 136(5):805-810. doi:10.1007/s00401-018-1913-0. Epub 2018 Sep 26

12. Brat DJ, Aldape K, Colman H (2020 Mar) cIMPACT-NOW update 5: recommended grading criteria and terminologies for IDH-mutant astrocytomas. Acta Neuropathol 139(3):603-608. doi:10.1007/s00401-02002127-9. Epub 2020 Jan 29

13. Tesileanu CMS, Dirven L, Wijnenga MMJ et al. Survival of diffuse astrocytic glioma, IDH1/2 wildtype, with molecular features of glioblastoma, WHO grade IV: a confirmation of the cIMPACT-NOW criteria. Neuro Oncol. 2020 Apr 15;22(4):515-523. doi: 10.1093/neuonc/noz200

14. Sahm F, Schrimpf D, Jones DT et al (2016 Jun) Next-generation sequencing in routine brain tumor diagnostics enables an integrated diagnosis and identifies actionable targets. Acta Neuropathol 131(6):903-910. doi:10.1007/s00401-015-1519-8. Epub 2015 Dec 15

15. Zacher A, Kaulich K, Stepanow S et al (2017 Mar) Molecular Diagnostics of Gliomas Using Next Generation Sequencing of a Glioma-Tailored Gene Panel. Brain Pathol 27(2):146-159. doi:10.1111/bpa.12367. Epub 2016 Apr 19

16. Capper D, Jones DTW, Sill M et al. DNA methylation-based classification of central nervous system tumours. Nature. 2018 Mar 22;555(7697):469-474. doi: 10.1038/nature26000. Epub 2018 Mar 14

17. Stichel D, Schrimpf D, Casalini B et al (2019 Nov) Routine RNA sequencing of formalin-fixed paraffinembedded specimens in neuropathology diagnostics identifies diagnostically and therapeutically relevant gene fusions. Acta Neuropathol 138(5):827-835. doi:10.1007/s00401-019-02039-3. Epub 2019 Jul 5

18. Singh B, Coffey RJ (2014) Trafficking of epidermal growth factor receptor ligands in polarized epithelial cells. Annu Rev Physiol 76:275-300

19. Mayer BJ (2015) The discovery of modular binding domains: building blocks of cell signalling. Nature Rev Mol Cell Biol 16:691-698

20. Thorpe LM, Yuzugullu H, Zhao JJ (2015) PI3K in cancer: divergent roles of isoforms, modes of activation and therapeutic targeting. Nat Rev Cancer 15:7-24

21. Verhaak RG, Hoadley KA, Purdom E et al.; Cancer Genome Atlas Research Network. Integrated genomic analysis identifies clinically relevant subtypes of glioblastoma characterized by abnormalities in PDGFRA, IDH1, EGFR, and NF1. Cancer Cell. 2010 Jan 19;17(1):98-110

22. Brennan CW, Verhaak RG, McKenna A et al.; TCGA Research Network. The somatic genomic landscape of glioblastoma. Cell. 2013 Oct 10;155(2):462-77

23. Furnari FB, Cloughesy TF, Cavenee WK et al (2015 May) Heterogeneity of epidermal growth factor receptor signalling networks in glioblastoma. Nat Rev Cancer 15(5):302-310

24. Eskilsson E, Rosland GV, Talasila KM et al (2016 Dec) EGFRvIII mutations can emerge as late and heterogenous events in glioblastoma development and promote angiogenesis through Src activation. Neuro

Page 15/22 
Oncol 18(12):1644-1655

25. An Z, Aksoy O, Zheng T et al (2018 March) Epidermal growth factor receptor (EGFR) and EGFRvIII in glioblastoma (GB): signaling pathways and targeted therapies. Oncogene 37(12):1561-1575

26. Villano JL, Mauer AM, Vokes EE (2003 Apr) A case study documenting the anticancer activity of ZD1839 (Iressa) in the brain. Ann Oncol 14(4):656-658. doi:10.1093/annonc/mdg153

27. Yang J, Yan J, Liu B (2017) Targeting EGFRvIll for glioblastoma multiforme. Cancer Lett 403:224-230. doi:10.1016/j.canlet.2017.06.024

28. Chi AS, Cahill DP, Reardon DA et al. Exploring Predictors of Response to Dacomitinib in EGFR-Amplified Recurrent Glioblastoma. JCO 130.238.007.040

29. Lee A, Arasaratnam M, Chan DLH et al (2020) Anti-epidermal growth factor receptor therapy for glioblastoma in adults. Cochrane Database Syst Rev. May 12;5(5):CD013238. doi:

10.1002/14651858.CD013238.pub2

30. Liu X, Chen X, Shi L et al. The third-generation EGFR inhibitor AZD9291 overcomes primary resistance by continuously blocking ERK signaling in glioblastoma. J Exp Clin Cancer Res. 2019 May 23;38(1):219. doi: 10.1186/s13046-019-1235-7

31. Chagoya G, Kwatra SG, Nanni CW et al. Efficacy of osimertinib against EGFRvIll + glioblastoma. Oncotarget. 2020 Jun 2;11(22):2074-2082. doi: 10.18632/oncotarget.27599

32. Luger AL, Lorenz NI, Urban H et al (2020) Activation of Epidermal Growth Factor Receptor Sensitizes Glioblastoma Cells to Hypoxia-Induced Cell Death. Cancers 12:2144. doi:10.3390/cancers12082144

33. Wen PY, Chang SM, Van den Bent MJ, ET AL. Response Assessment in Neuro-Oncology Clinical Trials. J Clin Oncol. 2017 Jul 20;35(21):2439-2449

34. World Medical Association. WMA Declaration of Helsinki: ethical principles for medical research involving human subjects. 64th WMA General Assembly, Fortaleza, Brazil (October 2013) Accessed November 30, 2020. https://www.wma.net/policies-post/wma-declaration-of-helsinki-ethical-principles-for-medicalresearch-involving-human-subjects/

35. Frampton GM, Fichtenholtz A, Otto GA et al (2013) Development and validation of a clinical cancer genomic profiling test based on massively parallel DNA sequencing. Nat Biotechnol 31:1023-1031

36. Palmisano WA, Divine KK, Saccomanno G et al (2000) Predicting lung cancer by detecting aberrant promoter methylation in sputum. Cancer Res 60:5954-5958

37. Yoshimoto K, Dang J, Zhu S et al Development of a real-time RT-PCR assay for detecting EGFRvIll in glioblastoma samples. Clin Cancer Res. 2008 14:488-493.; Idbaih A, Aimard J, Boisselier B et al. Epidermal growth factor receptor extracellular domain mutations in primary glioblastoma. Neuropathol Appl Neurobiol. 2009;35:208-213

38. Parsons DW, Jones S, Zhang X et al (2008) An integrated genomic analysis of human glioblastoma multiforme. Science 321:1807-1812

39. Ichimura K, Pearson DM, Kocialkowski S et al (2009) IDH1 mutations are present in the majority of common adult gliomas but rare in primary glioblastomas. Neuro-oncol 11:341-347

40. Taal W, Oosterkamp HM, Walenkamp AM et al (2014 Aug) Single-agent bevacizumab or lomustine versus a combination of bevacizumab plus lomustine in patients with recurrent glioblastoma (BELOB trial): a 
randomised controlled phase 2 trial. Lancet Oncol 15(9):943-953 doi: 10.1016/S1470-2045(14)70314-6. Epub 2014 Jul 15

41. Lombardi G, De Salvo GL, Brandes AA et al (2019 Jan;20(1):110-119) Regorafenib compared with lomustine in patients with relapsed glioblastoma (REGOMA): a multicentre, open-label, randomised, controlled, phase 2 trial. Lancet Oncol. doi: 10.1016/S1470-2045(18)30675-2. Epub 2018 Dec 3

42. Kesari S, Ram Z, EF-14 Trial Investigators (2017 Jul) Tumor-treating fields plus chemotherapy versus chemotherapy alone for glioblastoma at first recurrence: a post hoc analysis of the EF-14 trial. CNS Oncol 6(3):185-193. doi:10.2217/cns-2016-0049. Epub 2017 Apr 12

43. Chen JR, Xu HZ, Yao Y et al (2015 Nov) Prognostic value of epidermal growth factor receptor amplification and EGFRvIII in glioblastoma: meta-analysis. Acta Neurol Scand 132(5):310-322

44. Wang N, Zhang Y, Mi Y et al (2020 Sep) Osimertinib for EGFR-mutant lung cancer with central nervous system metastases: a meta-analysis and systematic review. Ann Palliat Med 9(5):3038-3047. doi:10.21037/apm-20-605. Epub 2020 Sep 14

45. Cross DA, Ashton SE, Ghiorghiu S et al (2014 Sep) AZD9291, an irreversible EGFR TKI, overcomes T790Mmediated resistance to EGFR inhibitors in lung cancer. Cancer Discov 4(9):1046-1061

46. Makhlin I, Salinas RD, Zhang D et al (2019) Clinical activity of the EGFR > tyrosine kinase inhibitor osimertinib in EGFR-mutant glioblastoma. CNS Oncol. Nov 1;8(3):CNS43

47. Lassman AB, Rossi MR, Raizer JJ et al. Molecular study of malignant gliomas treated with epidermal growth factor receptor inhibitors: tissue analysis from North American Brain Tumor Consortium Trials 0103 and 00-01. Clin Cancer Res. 2005 Nov 1; 11(21):7841-50

48. Mellinghoff IK, Wang MY, Vivanco I et al (2005 Nov) Molecular determinants of the response of glioblastomas to EGFR kinase inhibitors. N Engl J Med 10(19):2012-2024 353(

49. Schulte A, Liffers K, Kathagen A et al (2013 Oct) Erlotinib resistance in EGFR-amplified glioblastoma cells is associated with upregulation of EGFRvIll and PI3Kp1108. Neuro Oncol 15(10):1289-1301

50. Jun HJ, Acquaviva J, Chi D et al. Acquired MET expression confers resistance to EGFR inhibition in a mouse model of glioblastoma multiforme. Oncogene. 2012 Jun 21; 31(25):3039-50

51. Ma Y, Tang N, Thompson RC et al. InsR/IGF1R Pathway Mediates Resistance to EGFR Inhibitors in Glioblastoma. Clin Cancer Res. 2016 Apr 1; 22(7):1767-76

52. Chakravarti A, Loeffler JS, Dyson NJ. Insulin-like growth factor receptor I mediates resistance to antiepidermal growth factor receptor therapy in primary human glioblastoma cells through continued activation of phosphoinositide 3-kinase signaling. Cancer Res. 2002 Jan 1; 62(1):200-7

53. Luger AL, Lorenz NI, Urban H et al (2020) Activation of Epidermal Growth Factor Receptor Sensitizes Glioblastoma Cells to Hypoxia-Induced Cell Death. Cancers 12:2144. doi:10.3390/cancers12082144

54. He K, Qi Q, Chan CB et al (2013) Blockade of glioma proliferation through allosteric inhibition of JAK2. Sci Signal 6:ra55

55. de la Iglesia N, Konopka G, Puram SV et al (2008) Identification of a PTEN-regulated STAT3 brain tumor suppressor pathway. Genes Dev 22:449-462

56. Carro MS, Lim WK, Álvarez MJ et al (2010) The transcriptional network for mesenchymal transformation of brain tumours. Nature 463:318-325

Page $17 / 22$ 
57. Fan QW, Cheng C, Knight ZA et al (2009) EGFR signals to mTOR through PKC and independently of Akt in glioma. Sci Signal 2:ra4

58. Uht RM, Amos S, Martin PM et al (2007) The protein kinase C-eta isoform induces proliferation in glioblastoma cell lines through an ERK/Elk-1 pathway. Oncogene 26:2885-2893

59. Aeder SE, Martin PM, Soh JW et al (2004) PKC-eta mediates glioblastoma cell proliferation through the Akt and mTOR signaling pathways. Oncogene 23:9062-9069

60. Cvrljevic AN, Akhavan D, Wu M et al (2011) Activation of Src induces mitochondrial localisation of de27EGFR (EGFRvIII) in glioma cells: implications for glucose metabolism. Journal of Cell Sci 124:2938-2950

61. Struve N, Binder ZA, Stead LF et al (2020 Apr) EGFRvIII upregulates DNA mismatch repair resulting in increased temozolomide sensitivity of MGMT promoter methylated glioblastoma. Oncogene 39(15):30413055. doi:10.1038/s41388-020-1208-5. Epub 2020 Feb 17

62. Eskilsson E, Røsland GV, Solecki G et al (2018) EGFR heterogeneity and implications for therapeutic intervention in glioblastoma. Neuro Oncol. May 18;20(6):743-752. doi: 10.1093/neuonc/nox191

63. Weller M, Butowski N, Tran DD et al (2017 Oct) Rindopepimut with temozolomide for patients with newly diagnosed, EGFRvIII-expressing glioblastoma (ACT IV): a randomized, double-blind, international phase 3 trial. ACT IV trial investigators. Lancet Oncol 18(10):1373-1385

64. Lassman A, Pugh S, Wang TJ et al (2020) A randomized, double-blind, placebo-controlled phase 3 trial of depatuxizumab mafodotin (ABT-414) in epidermal growth factor receptor (EGFR) amplified (AMP) newly diagnosed glioblastoma (nGBM). Neuro-Oncology 21(Supplement_6):vi17-vi17

65. Santangelo A, Rossato M, Lombardi G et al. A molecular signature associated with prolonged survival in glioblastoma patients treated with regorafenib. Neuro Oncol. 2021 Feb 25;23(2):264-276. doi:

10.1093/neuonc/noaa156

\section{Supplemental Data}

Supplemental figures \& tables are not available with this version.

\section{Figures}




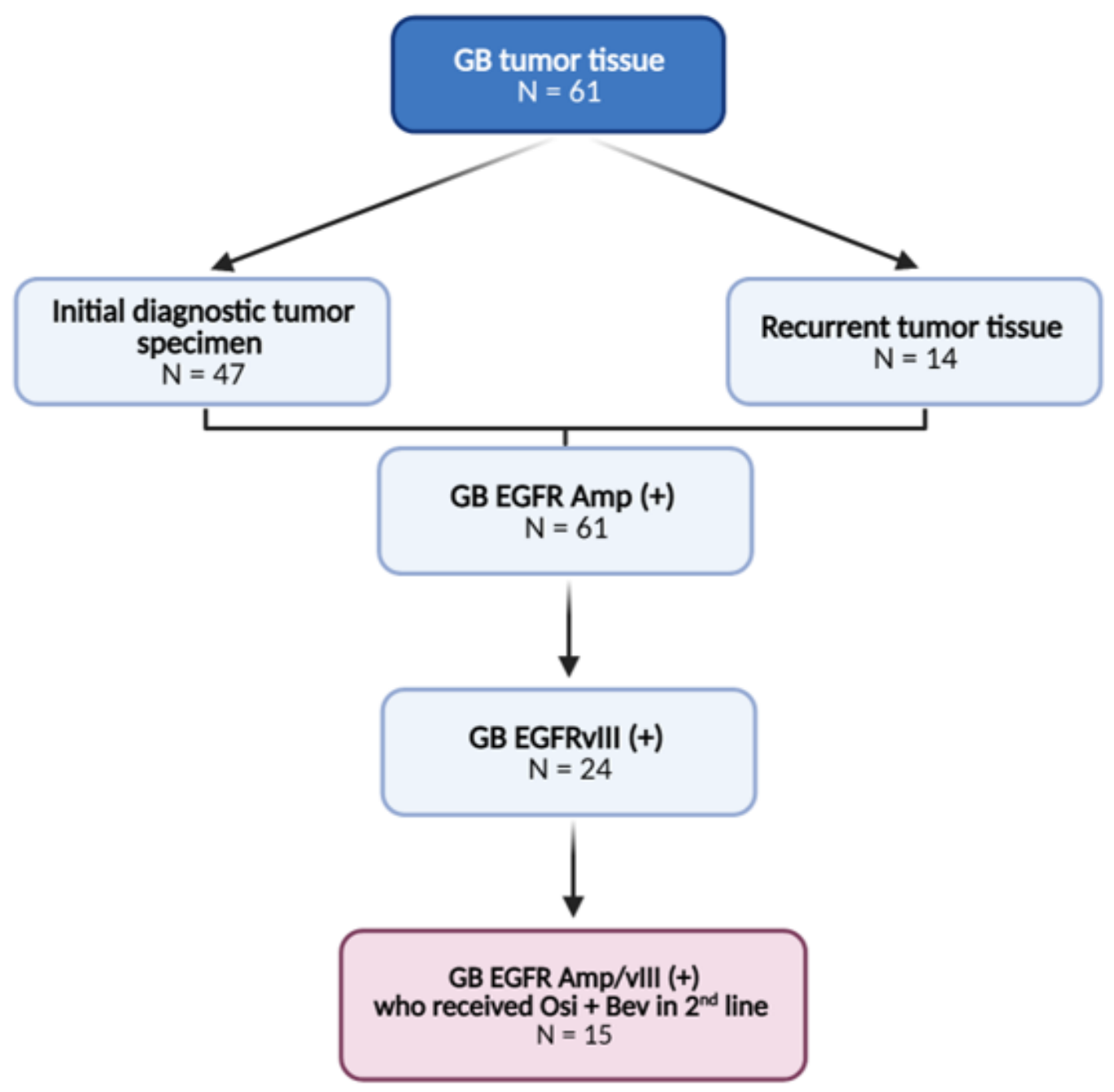

Figure 1

Flow of samples and patients who were tested for the presence of EGFR amplification and EGFRvIII mutation.

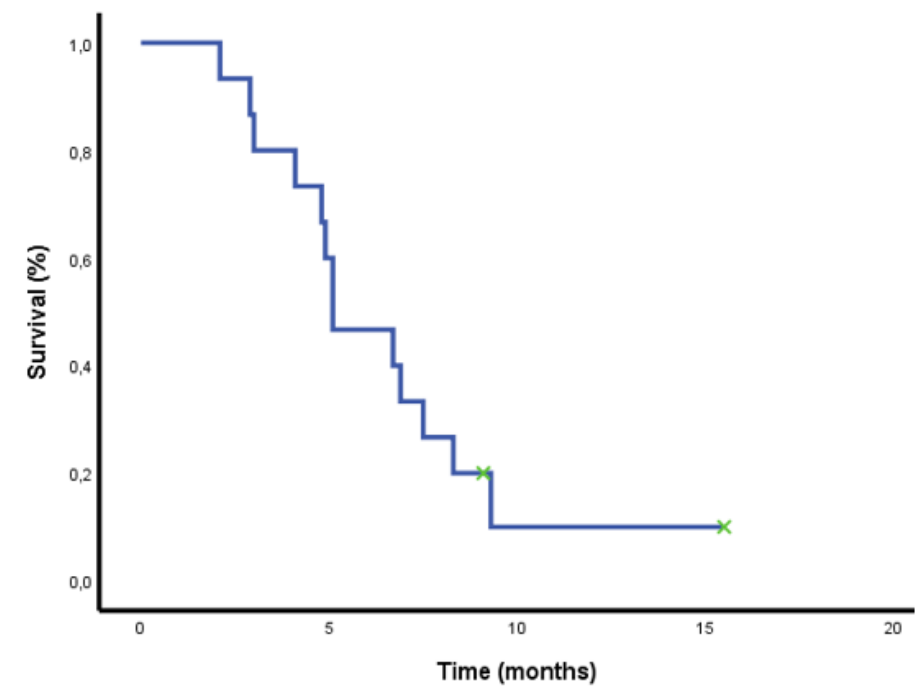

A

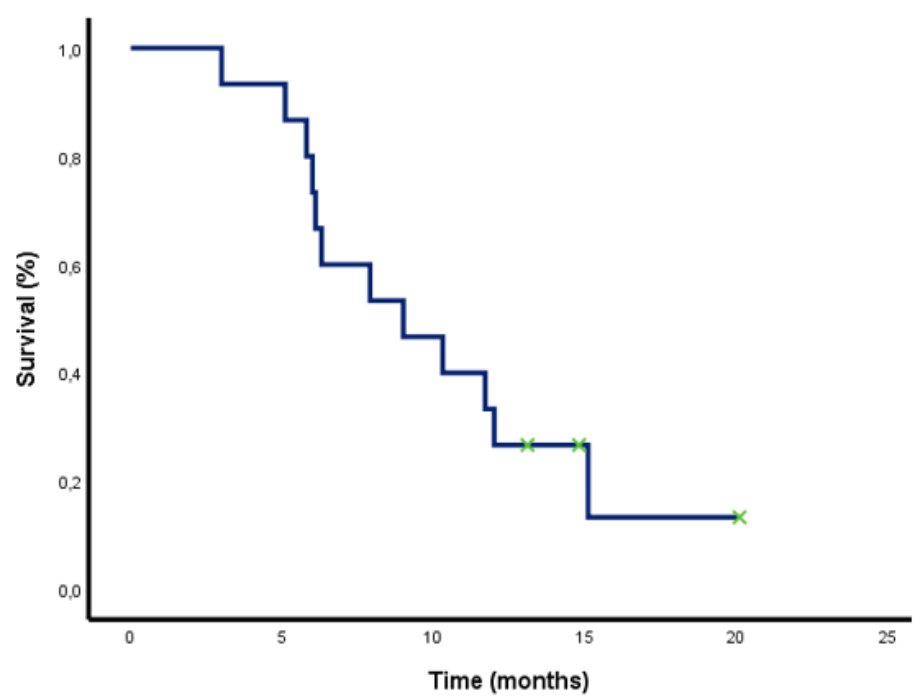

$\mathrm{B}$

Figure 2 
A. PFS to osimertinib/bevacizumab combination. B. OS to osimertinib/bevacizumab combination

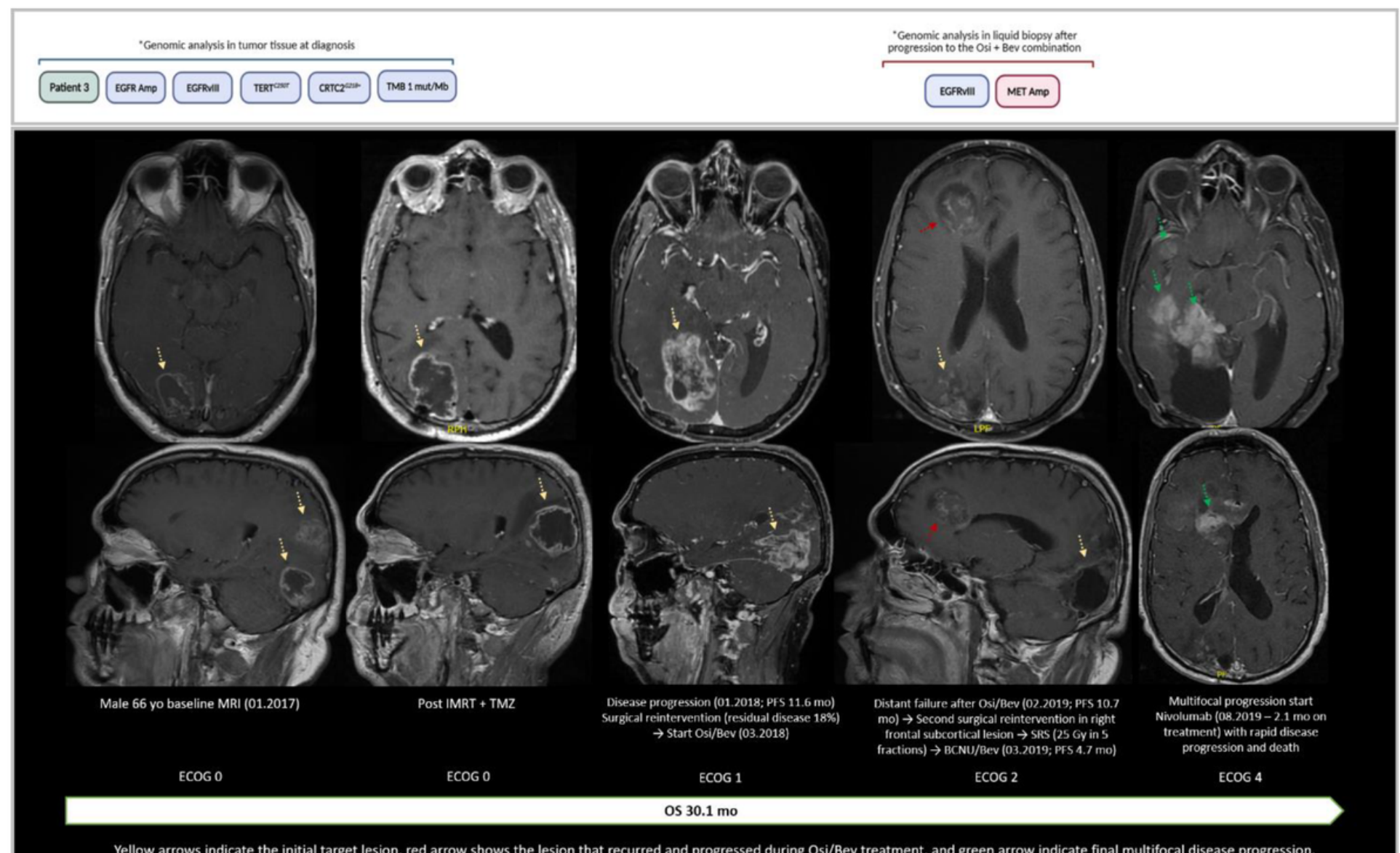

Yellow arrows indicate the initial target lesion, red arrow shows the lesion that recurred and progressed during Osi/Bev treatment, and green arrow indicate final multifocal disease progression.

\section{Figure 3}

Magnetic resonance imaging (MRI) scans of a representative case (patient \#3) who achieved a good response and long PFS after starting treatment with osimertinib/ bevacizumab.

* Genomic analysis in tumor tissue at diagnosis

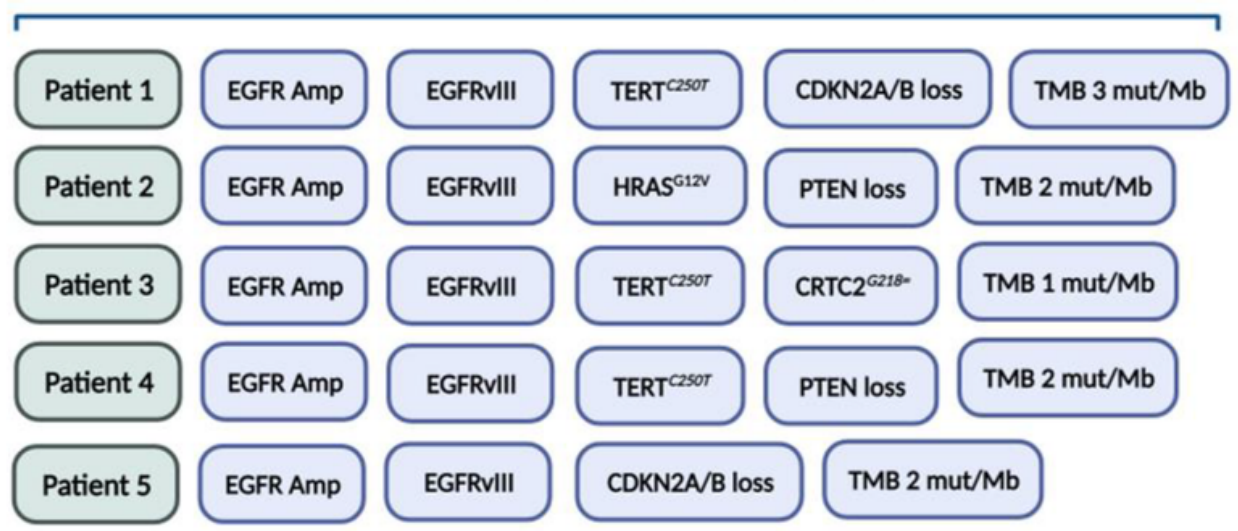

"Qualitative next generation sequencing that uses targeted high throughput hybridization-based capture technology (Foundation One and Foundation One liquid CDx')
*Genomic analysis in liquid biopsy after progression to the Osi + Bev combination

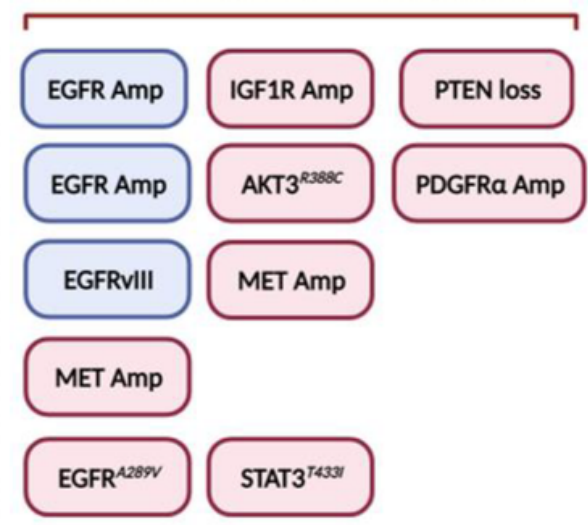

Figure 4 
Genomic findings in five selected patients comparing initial tissue and blood analysis after progression to osimertinib/bevacizumab.

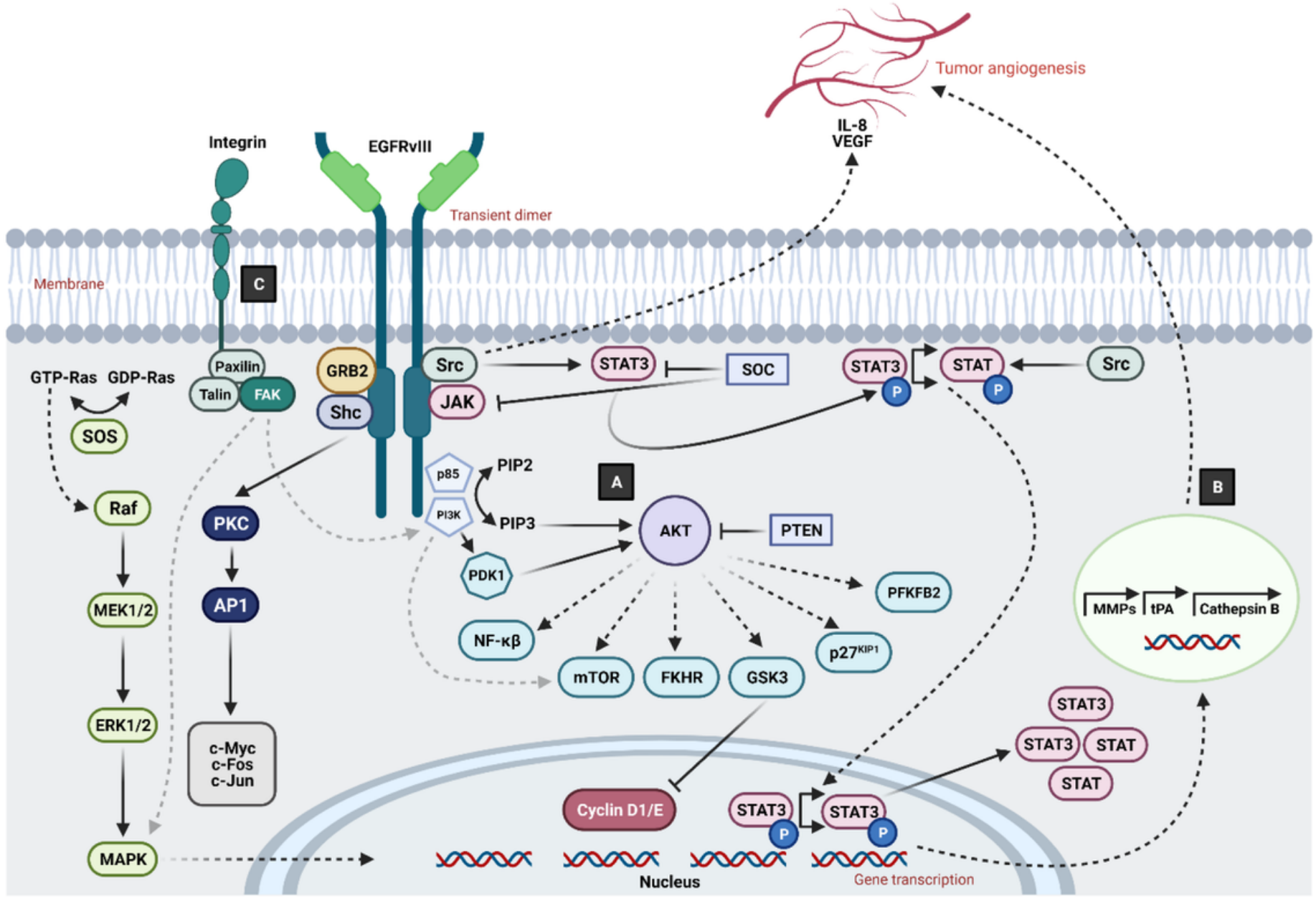

\section{Figure 5}

Visual representation of the signaling pathways activated by EGFR and EGFRvIII and their interaction with integrin, AKT/mTOR, and Wnt signaling. Activation upregulates different transcription factors involved in tumor cell proliferation, invasion, and angiogenesis, blocked by various EGFR- and EGFRvIll inhibitors. A. The PI3K/Akt pathway is known to control cell proliferation, growth, and apoptosis, but changes in this pathway can also increase cell invasion by inhibiting ECM detachment-induced cell death and stimulating MMP secretion.

Expression of EGFRvIII increases the activation of Akt through downregulation of the cell cycle inhibitor p27 and enhances cell proliferation. In vivo, EGFRvIll has a strong association with the phosphorylation of mTOR, and it has been demonstrated that the mutant receptor might be an activator of PI3K in the absence of PTEN loss. B. The Stat family of transcription factors acts as important signaling components and transfers the signal transduction of various cytokines and growth factors like EGF from the extracellular environment to the nucleus. Stat3 is upregulated in many cancer types and promotes immune responses and differentiation and cellular transformation, proliferation, and invasion. Upon activation of cell surface receptors through binding of its ligands, the receptor-associated Janus kinase (JAK) and the Src kinase act as connecting links between the receptors and Stat3. Stat3 is phosphorylated and translocated into the nucleus, where it induces the transcription of multiple genes involved in the regulation of cellular and tissue processes, including those that function in cell motility. The binding of EGF to EGFR leads to an activation of Stat3 and subsequently a Stat3- 
induced increased TWIST expression. Even though EGFR levels show a strong correlation with p-Stat3, the correlation of EGFRvIll with activated Stat3 seems to be even stronger. C. EGFRvIII in Integrin-FAK-ERK Signaling. It has been demonstrated that EGFRvIll constitutively activates the MAPK pathway in human glioma cells. Substrates of phosphatase and tensin homolog PTEN, a tumor suppressor in many cancer types, includes FAK and inhibits integrin- and growth factor-mediated MAPK signaling. PTEN phosphatase activity suppresses the invasion of EGFRvIll-expressing glioma cells. EGFRvIll could enhance the phosphorylation levels of FAK $n$ glioma cells while forming a complex, which correlates with the increased catalytic activity of FAK comparable to stimulation by growth factors or integrins. 